Revised Manuscript - changes highlighted in yellow

\title{
Surface stress sensors for rapid and ultrasensitive detection of active free drugs in human serum
}

Joseph W. Ndieyira ${ }^{1,2^{*}}$, Natascha Kappeler ${ }^{1}$, Stephen Logan ${ }^{1}$, Matthew A. Cooper ${ }^{3}$, Chris

Abell $^{4}$, Rachel A. McKendry ${ }^{{ }^{*}}$ \& Gabriel Aeppli ${ }^{1}$

1. London Centre for Nanotechnology, Division of Medicine and Department of Physics and

Astronomy

University College London

17-19 Gordon Street

London, WC1H 0AH

United Kingdom.

2. Jomo Kenyatta University of Agriculture and Technology

Department of Chemistry

Po Box 62000

Nairobi

Kenya.

3. Institute for Molecular Bioscience

The University of Queensland

Brisbane, 4072

Australia.

4. Department of Chemistry

Lensfield Road, University of Cambridge

Cambridge CB2 1EW 
United Kingdom

* Corresponding authors: Joseph Ndieyira Email: (j.ndieyira@ucl.ac.uk) and Rachel McKendry Email: (r.a.mckendry@ucl.ac.uk) 


\begin{abstract}
There is growing appreciation that mechanical signals can be as important as chemical and electrical signals in biology. To include such signals in a systems biology description for understanding pathobiology and developing therapies, quantitative experiments on how solution phase and surface chemistry together produce biologically relevant mechanical signals are needed. Due to the appearance of drug-resistance hospital "superbugs", a system of large current interest is the destruction of bacteria by antibiotics forming bound drug/target complexes which stress the bacterial cell membranes. Here we use nanomechanical cantilevers as surface stress sensors together with equilibrium theory to describe quantitatively the mechanical response of a surface receptor to different antibiotics in the presence of competing ligands in solution. The antibiotics examined are the standard, FDA approved drug of last resort, vancomycin, as well as yet-to-be approved oritavancin, which shows promise for controlling vancomycin resistant infections. The work reveals variations among strong and weak competing ligands, such as proteins in human serum, which determine dosages in drug therapies. These findings further enhance our understanding of the biophysical mode of action of the antibiotics and will help develop better treatments, including choice of drugs as well as dosages, against pathogens.
\end{abstract}


Biochemical systems, such as hormones and transmitters, generate signals in target cells by interacting with surface receptors. When an effector molecule and a surface receptor combine, a new structural entity is formed ${ }^{1}$, and the associated stress provides a mechanical signal for subsequent biological activity. For example, the antibiotic vancomycin (Van) binds to bacterial cell wall precursors to disrupt membrane integrity, effectively stopping the bacteria from forming a rigid network of peptides on the cell surface ${ }^{2,3}$. Competing molecules in solution can affect such binding: in antimicrobial therapy, serum proteins are inhibitors which reduce the efficacy of drugs $^{4,5}$ while the competing soluble ligands such as diacetyl Lysine-D-Alanine-D-Alanine or diacetyl vancomycin susceptible receptors (Ac-VSR), an analogue to the bacterial cell wall peptide precursors ${ }^{6}$ are thought to enhance the ability of drugs to inhibit bacterial cell growth in a clinical setting ${ }^{7}$.

Serum proteins bind to drugs in blood and in so doing reduce the free concentration of active drug and its penetration into cell tissues. Accordingly, a drug that is bound to blood serum is no longer available to target the pathogenic organisms and so it is antibacterially inactive. Thus, quantifying active free drug concentrations in comparison to the total inhibitory concentration administered is important for determining the correct and safe patient dosage. Furthermore, understanding the full impact of competitive ligands on the activity of selective inhibitors is important for the design of effective drug molecules to control infections as well as for determining dose levels. Even so, we are unaware of experiments exploring how interactions between competing inhibitory ligands and drug molecules can alter the mechanical properties of surface bound receptors to regulate the efficacy of drugs.

Here we describe a new methodology to monitor the interactions of molecules in solution and their impact on the surface mechanics of receptors to evaluate drug action in a competitive 
environment. We formulate a mathematical model to quantify surface stress changes where the reactions are occurring in solution and at the surfaces (Fig. 1). As depicted in Figure 1, we performed assays without physically separating bound from unbound drugs, which is more representative of the physiological environment. We propose the hypothesis that interaction between a selective inhibitor and competing ligand in solution affects surface action, which controls pharmacological efficacy. We test this hypothesis on two powerful antibiotics; Van as a model compound and oritavancin (Ori) as a new drug, respectively. Van has been used extensively in tissue engineering and in controlled drug release studies ${ }^{8}$. It is currently in clinical use as one of the last powerful antibiotics against methicillin-resistant Staphylococcus aureus (MRSA) as well as Clostridium difficile infections (CDI), and, indeed the recently identified multidrug-resistant clones of $\mathrm{MRSA}^{9}$. In contrast, Ori is a semi-synthetic lipoglycopeptide in clinical development against serious gram-positive infections caused by $\operatorname{MRSA}^{10}$.

\section{Nanomechanical Detection of Ligand-Receptor Interactions}

To measure ligand-receptor interactions necessary to generate changes in surface stress and to decouple chemistry from surface mechanics, we selected a cantilever technique. As a stress sensor of surface bound receptors, the cantilever technology is unrivalled. All conventional tests such as filter binding assays ${ }^{11}$, equilibrium dialysis ${ }^{12}$, fluorescence polarisation immunoassay $(\text { FPIA })^{13}$ or surface plasmon resonance $(\mathrm{SPR})^{14}$ measure optical signatures of chemical binding, which do not yield the most important property for mechanical signalling, namely surface stress. Cantilevers, with subnanometer sensitivity ${ }^{14-17}$ allow direct monitoring and simultaneous quantitation of solution and surface reactions in a

single step where specificity is achieved by using in-situ referencing ${ }^{15-18}$. The reactions are 
quantified by tracking stress changes induced from the ligand-receptor interactions causing cantilever bending deflections, measured optically ${ }^{15}$ or electrically ${ }^{18}$.

To probe surface stresses due to drug-target interactions, arrays of cantilevers were functionalised with surface biomimetic capture molecules, namely vancomycin-susceptible receptors (or $\boldsymbol{V S R}$ ), analogues to bacterial cell wall precursors that present un-cross-linked peptide motifs terminating in the sequence Lysine-D-Alanine-D- Alanine ${ }^{6,19,20}$. Artefacts that produce non-specific signals were overcome by carrying out differential measurements where we subtracted reference mercaptoundecyl tri(ethylene glycol) thiol (PEG)-coated cantilever bending signals from the $\boldsymbol{V S R}$ signals. The bio-specific binding efficiency was further enhanced by the passivation of the "bottom" surface of the cantilevers using $\boldsymbol{P E \boldsymbol { E } \text { - }}$ silane (Supplementary information).

\section{Impact of Weak Competing Ligands on the Efficacy of Drugs}

Serum proteins, a vital component of plasma in the transport of drugs, lipids or steroid hormones bind weakly to antibiotics ${ }^{21}$ and display strong inhibitory effects ${ }^{4,5}$ and therefore serve as a good model for investigating the impact of weak competing ligands. Figure 2 shows the differential bending signals obtained after injection of blood serum and Polysorbate 80 (PS80) $^{22}$, as well as after injection of $3 \mu \mathrm{M}$ Van and $0.5 \mu \mathrm{M}$ Ori. Remarkably, antibiotics were detected even in $98 \%$ whole blood serum (Fig. $2 \mathrm{~b}$ and c). The increasing noise with serum concentrations observed in our measurements was probably caused by the scattering of the laser light by serum proteins. Furthermore, as the serum concentration increases, the differential stress signals decrease because of the binding of drug molecules to blood serum. The cantilever deflection signal is proportional to the quantity of active free drug in the physiological medium, so the bending response is a measure of the effectiveness 
of dosage and correspondingly, a realistic in-vitro susceptibility test for the drugs. We thus can directly quantitate mechanically the effectiveness of dosages without prior amplification steps (for example FPIA). For this purpose, our approach unambiguously identifies the deflections caused by specific interactions between drugs in blood serum and the surface receptors yielding the first experimental evidence that drug-serum complexes only reduce surface stress (Fig. 2b-c).

In pharmacology, the potency of drugs is often quoted as $I C_{50}$, the concentration of antagonist that gives rise to $50 \%$ inhibition of the activity of a selective inhibitor ${ }^{23}$. To quantify the $I C_{50}$ of drug-serum interactions, a wider range of serum concentrations in the presence of all antibiotics were investigated and the results of the equilibrium differential surface stress summarised in Figure 2d. Equation (1), whose detailed derivation is given in the Supplementary information, was used to calculate $I C_{50}$ values:

$$
\Delta \sigma_{e q}=\Delta \sigma_{\min }+\frac{\sigma_{\max }-\sigma_{\min }}{1+\frac{\left[\text { ligand }_{2}\right]}{I C_{50}}}
$$

where $\sigma_{\text {eq }}$ corresponds to equilibrium surface stress, $\sigma_{\min }$ is the constant of proportionality which corresponds to the minimum surface stress generated by a selective inhibitor (ligand 1 ) in the presence of large excess of the concentration of competitive ligand (ligand $)_{2}$ and $\sigma_{\max }$ is the constant of proportionality which corresponds to the maximum surface stress generated by ligand $d_{1}$ in the absence of ligand $d_{2}$. The analysis shown in Figure $2 \mathrm{~d}$ and in Table $\mathrm{S} 1$ is the outcome of the fit of equation (1) superposed onto measured antibiotic differential stress signals revealing $I C_{50}$ values in whole serum; $700 \pm 100 \mu \mathrm{M}$ for $\boldsymbol{V a n}$ and $408 \pm 70 \mu \mathrm{M}$ for 
Ori. Although Ori's dose is 6 times lower than Van, a relatively higher concentration of serum proteins was required to inhibit its surface stress on the cantilever.

\section{Impact of Strong Competing Ligands on the Efficacy of Drugs}

To further examine the role of competing soluble ligands on the surface functionality of drugs, we utilised Ac-VSR because of its strong $\mu \mathrm{M}$ interactions with antibiotics in solution $^{6,19,20}$. Again, the inhibitory effect was quantified by varying the concentrations of the soluble ligand while keeping the total antibiotic concentrations $[\boldsymbol{V a n}]$ and $[\boldsymbol{O r i}]$ constant at 50 $\mu \mathrm{M}$ and $0.1 \mu \mathrm{M}$ respectively. These particular antibiotic concentrations were chosen because: i) they give relatively large mechanical bending signals, ii) are found to fall within the linear portions of the Langmuir Adsorption Isotherm, and iii) are readily inhibited by adding reasonable concentrations of the solubilised ligands. Figure 3 shows the outcome of drugtarget binding interactions in the presence of Ac-VSR. Our measurements reveal that for 500 $\mu \mathrm{M}$ competing ligand concentration, the interactions of all antibiotics at a surface were $40 \%$ inhibited despite having intial doses differing by a factor of 500 . The equilibrium surface stress at each antibiotic concentration was measured on at least 4 arrays, and the resulting stress data sets were then modeled by equation (1), yielding $I C_{50}$ of $110 \pm 10 \mu \mathrm{M}$ for Van and $400 \pm 70 \mu \mathrm{M}$ for $\boldsymbol{O r i}$ respectively. The enhanced $I C_{50}$ values observed in the presence of analogous competing ligand to the surface receptor is not surprising given that in a whole bacteria assay, a large amount of inhibitory ligand ${ }^{24}$ is required to induce complete loss of activity (Supplementary Table S2).

Figure 4 shows that for lower antibiotic concentrations $(100 \mathrm{pM}-0.1 \mu \mathrm{M})$, particularly of oritavancin, $50-60$ min was required to reach thermodynamic pseudo-equilibrium. In contrast, for higher antibiotic concentrations $(0.5 \mu \mathrm{M}-10 \mu \mathrm{M})$, equilibrium was reached in 
just under 3 minutes. Our experiments show that cantilever detection sensitivity is governed to a large extent by the degree of the affinity/avidity of the receptor-ligand interactions. For example, for the antibiotic drug vancomycin, which binds predominantly as a monomer, the minimum cantilever-detectable drug concentration is $10 \mathrm{nM}^{15}$. This is 30 times more sensitive than established approaches such as the commercially available SPR method, for which the detection of $300 \mathrm{nM}$ vancomycin has been reported (Table 1). To demonstrate the ultimate sensitivity and to provide the evidence for our hypothesis that affinity/avidity determines detection sensitivity, we measured the binding of oritavancin, a novel antibiotic, against the same surface capture receptor as vancomycin. The data reveal a detection sensitivity of $100 \mathrm{pM}$ antibiotic concentration in buffer (Fig. 4), which is two orders of magnitude lower than for vancomycin ${ }^{15}$.

Figure $5 \mathrm{a} \& \mathrm{~b}$ show the equilibrium differential bending signals obtained from blood serum concentration fixed at $98 \%$ after injection of different concentrations of antibiotics. The minimal response of $i n$-situ reference $\boldsymbol{P E} \boldsymbol{G}$ cantilevers, which is anti-adsorbative, even for very high antibiotic concentrations provides strong evidence of surface specific binding to $\boldsymbol{V S R}$. Moreover, to compare the variations in the magnitude of surface stress signals within each antibiotic and from across antibiotics (Fig. 2-5), we plotted cantilever bending deflection signals over the same time scales of 40 mins.

\section{Modelling the System of Antibiotic, Solvent and Surface Activity}

To quantify the correlation between solution matrix and surface mechanics (Supplementary information), we began by considering model (I) shown in Figure 1a where the stress induced in surface bound receptors in the presence of ligand $_{1}$ scales as a function of ligand concentration. The changes in surface mechanics are monitored by the expression: 


$$
\Delta \sigma_{1}=\frac{\sigma_{\max }\left[\text { ligand }_{1}\right]^{n}}{K_{1}^{n}+\left[\text { ligand }_{1}\right]^{n}}
$$

where $\sigma_{1}$ corresponds to surface stress, $\boldsymbol{n}$ is the stoichiometric coefficient of the reactions without a competing soluble ligand and $K_{1}$ is the surface thermodynamic equilibrium dissociation constant. It is raised to the power $\boldsymbol{n}$ to ensure that $K_{1}$ maintains the dimension of concentration as $\boldsymbol{n}$ varies. Setting $\boldsymbol{n}=1$ yields the Langmuir isotherm where the reactions occur independently, while $\boldsymbol{n}>1$ corresponds to positive co-operative interactions and $\boldsymbol{n}<1$ is a measure of negative co-operativity. While equation (2) yields the key parameters $K_{1}$ and $\sigma_{\max }$, responsible for quantifying surface binding interactions and the mechanical properties, it cannot quantify free ligand concentrations in solution, essential for biological activity. Thus we devised a second model (II), illustrated in Figure 1b, where we consider the simultaneous interactions, whose strength is given by $K_{3}$, between surface bound receptors and multiplexed soluble ligands. Again, the changes in surface mechanics induced by the reactions with competing ligand $_{2}$ in solution and at the surface targets are quantified by the expression, (see Supplementary information) where the solution phase stoichiometric coefficient $\boldsymbol{m}=1$, the system volume is infinite, and [ligand 2$]>>\left[\right.$ ligand $\left._{1}\right]$ :

$$
\sigma_{2}=\frac{\sigma_{\max }\left[\text { ligand }_{1}\right]^{n}}{K_{1}^{n}\left(1+\left(\frac{\left[\text { ligand }_{2}\right]}{K_{3}}\right)^{n}\right)+\left[\text { ligand }_{1}\right]^{n}}
$$

where $\sigma_{2}$ corresponds to surface stress in the presence of competing ligands and $K_{3}$ is the solution thermodynamic equilibrium dissociation constant. Eq. (3), which reduces to Eq. (1) if the concentration of [ligand 1 ] is fixed and $\boldsymbol{n}=1$, offers a particular understanding of ligand-receptor interactions and may help to design better assays to detect activation or 
inhibition of complex signal transduction pathways in response to selective inhibitors or treatments. The difference between models (I) and (II) is that in the latter case we account for the concentration of free drug molecules in a physiological medium which governs their activity via competitive serum. Accordingly, we modeled our stress data using equation (3) as a description of ligand-receptor interactions to quantify simultaneous surface and solution reactions. The resulting analysis allows us to evaluate the impact of serum binding on the concentration of active free drugs (Fig. $5 \mathrm{c} \&$ d). The parameters $\sigma_{\max }, m, K_{1}$ and $K_{3}$, which characterise the surface mechanics and solution matrix are coupled in a statistical sense. Therefore, to determine $K_{3}$, we first carried out a nonlinear least-square best fit of equation (3) to the antibiotic stress data from phosphate buffer, i.e. in the absence of serum proteins. This was achieved by setting $\left[\right.$ ligand $\left.d_{2}\right]=0$. The maximum surface stress signals, $\sigma_{\max }$ generated by $\boldsymbol{V a n}$ and $\boldsymbol{O} \boldsymbol{r}$ i were found to be $27 \pm 3 \mathrm{mN} / \mathrm{m}$ and $36.8 \pm 3.8 \mathrm{mN} / \mathrm{m}$ respectively. The $K_{1}$ in phosphate buffer was calculated as $0.5 \pm 0.2 \mu \mathrm{M}$ for $\boldsymbol{V a n}$ and $0.04 \pm 0.1 \mu \mathrm{M}$ for $\boldsymbol{O r i}$ while the stoichiometric coefficients values were $\boldsymbol{n}=1$ for $\boldsymbol{V a n}$ and $\boldsymbol{n}=1.2 \pm 0.2$ for $\boldsymbol{O r} \boldsymbol{i}$ respectively. These binding constants from the cantilever measurements are in good agreement with other sensor methods, for example SPR (Table 1).

Inspection of the denominator of Eq. (3) shows that the effect of serum on the nanomechanics of surface drug-target interactions is entirely via the term $\left(K_{1} / K_{3}\right)$ [serum]; in this case ligand $_{2}=$ serum. We therefore repeated the process of least-square best fits of Eq. (3) to the antibiotic stress data in the presence of serum proteins, where the parameters $\sigma_{\max }, \boldsymbol{n}$, and $K_{1}$ were fixed to their respective values in phosphate buffer while the serum concentration was fixed at $\sim 98 \%$. The $K_{3}$ for $\boldsymbol{V a n}$ binding to serum proteins in solution was found to be 500 $\pm 90 \mu \mathrm{M}, 2000$ times weaker than for Ori where $K_{3}$ is $30 \pm 6 \mu \mathrm{M}$. Supplementary Table S1 shows a summary of $K_{3}$ for solution phase interactions via equations (1) and (4) in different 
media and makes comparison with direct mechanical quantitation, where the $K_{3}$ values observed in whole serum, within experimental error are in good agreement, validating equation (3). The fact that $\sigma_{\max }$ and stoichiometric coefficients are the same in buffer and serum makes it unlikely that serum-drug complexes bind surface bacterial cell wall targets. Supplementary Table S2 shows a summary of $K_{1}$ for surface interactions in different media and makes comparison with reported Minimum Inhibition Concentration (MIC) values from whole bacterial assays ${ }^{28}$, where the MICs' observed in whole serum are in good agreement with the $K_{1}$ 's from cantilever measurements.

The chemical equilibrium equations for the model of Fig. $1 \mathrm{~b}$ can also be solved analytically (Supplementary information) to yield an expression for the fraction $f_{b}$ of drugs bound to serum proteins:

$$
\begin{aligned}
& f_{b}=\frac{a+\sqrt{a^{2}-4 b}}{2[\text { drug }]} \\
& a=\left(K_{3}+[\text { drug }]+[\text { serum }]\right) \\
& b=([\text { drug }]+[\text { serum }])
\end{aligned}
$$

Using Eq. (4), our results for $K_{3}$ together with $[$ Van $]=3 \mu \mathrm{M},[$ Ori $]=0.5 \mu \mathrm{M}$ and $[$ serum $]=$ $98 \%$ result in $\sim 54 \%$ bound for $\operatorname{Van}$ and $\sim 95 \%$ bound for Ori. This is in agreement with previous work where the proportion of $\boldsymbol{V a n}$-serum complex in the literature averages $55 \%$ in humans, although a wide range (10 to $80 \%$ bound) has been reported ${ }^{21}$ while the effective amount of Ori bound to serum proteins varies between 50 and $95 \%{ }^{29}$. Equilibrium dialysis and related techniques have been the methods of choice to study blood-serum interactions but are subject to errors ${ }^{30}$, limiting their accuracy at clinically relevant antibiotic doses which stand at $(3-27 \mu \mathrm{M})^{31}$. In contrast, the mechanical quantitation of efficacious drug 
concentration in blood serum is not limited by such errors and by using the low end of therapeutic antibiotic doses of $3 \mu \mathrm{M}$ Van and $0.5 \mu \mathrm{M}$ Ori, within the clinically relevant therapeutic window $(3-27 \mu \mathrm{M})^{31}$ as administered in hospitals, we find that the active free drug concentrations are $\sim 1.4 \mu \mathrm{M}$ Van and $\sim 0.1 \mu \mathrm{M}$ Ori. The reported MICs in whole serum for $\boldsymbol{V a n}$ is $\sim 0.7 \mu \mathrm{M}$ and for $\boldsymbol{O r i}$, it is $\sim 1 \mu \mathrm{M}$ for most staphylococci ${ }^{28}$, and the agreement with our cantilever-based measurements (Supplementary Table S2) demonstrates convincingly that surface mechanics is strongly linked to the efficacy of drugs.

\section{Novel Interactions of Bound Complexes in Solvents with Surface Receptors}

For serum in solution, the agreement of cantilever data over the full range of antibiotic and serum concentrations with Eq. (3) indicates the validity of the model in Fig. 1b with the rate constants $K_{2}$ and $K_{4}$ neglected. We show now that when we replace serum proteins with small peptides, this simplification matches the actions of vancomycin but not for oritavancin. Here we take advantage of the fact that direct comparison of equations (1) and (3) for $\boldsymbol{m}=1$ yields the conventional Cheng-Prusoff equation ${ }^{23}$ :

$I C_{50}=K_{3}+\frac{K_{3}}{K_{1}}\left(\left[\right.\right.$ ligand $\left.\left._{1}\right]\right)$

If $K_{3}$ and $I C_{50}$ are known, equation (5) can be used to characterise the surface binding affinity of selective inhibitors in a competitive environment. Accordingly, we estimated the $K_{1}$ values for drug binding to surface receptors in the presence of analogues Ac-VSR using $K_{3}$ for $\boldsymbol{V a n}$ $\sim 1 \mu \mathrm{M}^{6}$ and $\sim 3.8 \mu \mathrm{M}^{20}$ for $\boldsymbol{O r i}$; our estimate of $K_{1}$ for $\boldsymbol{V a n}$ is $\sim 0.46 \pm 0.02 \mu \mathrm{M}$ and $\sim(9.6$ $\pm 2.4) \times 10^{-4} \mu \mathrm{M}$ for $\boldsymbol{O r i}$. The $K_{1}$ for $\boldsymbol{V a n}$ is indistinguishable from that measured by varying $[\boldsymbol{V a n}]$ in phosphate buffer while for Ori it is a remarkable 50 times smaller than in phosphate buffer, yielding the lowest known dissociation constant for a receptor-drug binding 
interaction involving a small drug molecule. To test the accuracy of these values, we substituted Eq. (4) into Eq. (1) where the $K_{1}$ was fixed to its respective value in phosphate buffer to calculate the expected nano-mechanical stress. The results are plotted as dashed lines in Figure $3 \mathrm{c} \& \mathrm{~d}$ for comparison to the solid lines where Eq. (1) with $K_{1}$ was allowed to vary. The comparison validates the conclusion that there is nothing unusual about vancomycin, where the solid and dashed lines are negligibly different, while roughly an order of magnitude leftward shift for oritavancin confirms the widely differing $K_{1}$ 's obtained indirectly from Eq. (5) and directly from varying [Ori] in phosphate buffer in the absence of competing Ac- $\boldsymbol{V S \boldsymbol { R }}$ ligands. The implication is that for oritavancin and Ac- $\boldsymbol{V S \boldsymbol { R }}$ in solution, the pathways labeled by $K_{2}$ and $K_{4}$ in Fig $1 \mathrm{~b}$ cannot be neglected, meaning that in the presence of these ligands, the complexes bound in solution can also bind to membrane surface targets and therefore induce stress.

\section{Conclusions}

Our comprehensive experimental study at clinically relevant antibiotic drug doses (3-27 $\mu \mathrm{M})^{31}$ of the transduction between chemical and mechanical signals has uncovered the role of strong and weak competing ligands in the functionality of drugs and shows quantitatively how surface binding affinity is correlated with competing solution phase processes ${ }^{32,33}$. It represents to our knowledge the first use of nanomechanics for solving a practical pharmacological problem, namely that of comparing a novel drug such as oritavancin, with an older drug, vancomycin, threatened by evolving antimicrobial resistance. Beyond providing surface binding constants, our in-vitro competitive inhibition assays provide guidance and understanding as to the therapeutic doses needed in whole blood. More generally, we have shown that by combining classical chemical equilibrium theory with 
nanomechanical measurements, we can produce a compact quantitative description of a basic biological system involving mechanical events and multiple chemical pathways.

The experiments demonstrate that distinct changes of surface mechanics are drug-specific and that the solvent matrix plays a fundamental role in modulating the surface mechanics via straightforward inhibition due to competitive binding. It is clear that direct mechanical quantitation of the effectiveness of dosages using equation (3) without prior amplification steps will lead to progress in pharmacology and new insights into optimal clinical regimes, such as combination therapies. The experiments provide a blueprint for further studies to determine the role of chemistry and mechanics in membrane-bound receptors and proteins, which in turn will lead to the design of both better drugs as well as diagnostic platforms based on surface sensing layers. Perhaps the most obvious diagnostic application is to measure the active free drug availability in blood for a particular medical target and thereby determine appropriate doses tailored for individualised patients. With suitably engineered surface probes such as miniaturised cantilever arrays in a flow-through geometry, the diagnostic device would be paired with customised drug delivery for anaesthesia, anti-cancer, anti-HIV and antibacterial therapies. 
Additional Information

Supplementary information accompanies this paper at www.nature.com/naturenanotechnology Reprints and permission information is available online at http://npg.nature.com/reprints and permissions/. Correspondence and requests for materials should be addressed to JWN and RAM. 


\section{REFERENCES}

(1) Ghatkesar, M. K., Lang, H-P., Gerber, C., Hegner, M. \& Braun, T. Comprehensive characterization of molecular interactions based on nanomechanics. PLOS ONE. 3, 1-6 (2008).

(2) Dwyer, D. J. et al. Antibiotic-induced bacterial cell death exhibits physiological and biochemical hallmarks of apoptosis. Molecular Cell 46, 561-572 (2012).

(3) Kohanski, M. A., Dwyer, D. J., Wierzbowski, J., Cottarel, G. \& Collins, J. J. Mistranslation of membrane proteins and two-component system activation trigger antibiotic-mediated cell death. Cell 135, 679-690 (2008).

(4) Zeitlinger, M. A. et al. Protein binding: Do we ever learn? Antimicrob. Agents Chemother 55, 3067-3074 (2011).

(5) Bailey, E. M., Rybak, M. J. \& Kaatz, G. W. Comparative effect of protein binding on the killing activities of teicoplanin and vancomycin. Antimicrob. Agents Chemother 35, 1089-1092 (1991).

(6) Perkins, H. R. Specificity of combination between mucopeptide precursors and vancomycin or ristomycin. Biochem. J. 111, 195-205 (1969).

(7) Beauregard, D. A., Williams, D. H., Gwynn, M. N. \& Knowles. D. J. Dimerization and membrane anchors in extracellular targeting of vancomycin group antibiotics. Antimicrob. Agents Chemother. 39, 781-785 (1995).

(8) Lai, C-Y. et al. A mesoporous silica nanosphere-based carrier system with chemically removable $\mathrm{CdS}$ nanoparticle caps for stimuli-responsive controlled release of neurotransmitters and drug molecules. J. Am. Chem. Soc. 125, 4451-4459 (2003).

(9) Sieradzki, K. et al. The development of vancomycin resistance in a patient with methicillin-resistant Staphylococcus aureus infection. The New England Journal of Medicine 340, 517-523 (1999). 
(10) McKay, G. A et al. Time-kill kinetics of oritavancin and comparator agents against staphylococcus aureus, enterococcus faecalis and enterococcus faecium. $J$. Antimicrob. Chemother. 63, 1191-1199 (2009).

(11) Minamide, L. S. \& Bamburg, J. R. Filter paper dye-binding assay for quantitative determination of protein without interference from reducing agents or detergents. Anal. Biochem. 190, 66-70 (1990).

(12) Lonnroth, P., Jansson, P. A. \& Smith, U. A microdialysis method allowing characterization of intercellular water space in humans. AJP. 253, E228-E231 (1987).

(13) Nasir, M. S. \& Jolley, M. E. Fluorescence polarization: an analytical tool for immunoassay and drug discovery. Combinatorial chemistry \& high throughput screening. 2, 177-190 (1999).

(14) Frostell-Karlsson, A. et al. Biosensor analysis of the interaction between immobilised human serum albumin and drug compounds for prediction of human serum albumin binding levels. J. Med. Chem. 43, 1986-1992 (2000).

(15) Ndieyira, J. W. et al. Nanomechanical detection of antibiotic-mucopeptide binding in a model for superbug drug resistance. Nature Nanotech. 3, 691-696 (2008).

(16) Arlett, J. L., Myers, E.B. \& Roukes, M. L. Comparative advantages of mechanical biosensors. Nature Nanotech. 6, 203-215 (2011).

(17) Fritz, J. et al. Translating biomolecular recognition into nanomechanics. Science 288, 316-318 (2000).

(18) Shekhawat, G., Tark, S-H. \& Dravid, V. P. MOSFET-Embedded microcantilevers for measuring deflection in biomolecular sensors. Science 311, 1592-1595 (2006).

(19) Williams, D. H. Maguire, A. J. Tsuzuki, W. \& Westwell, M. S. An analysis of the origins of a cooperative binding energy of dimerisation. Science 280, 711-714 (1998). 
(20) Allen N. E. \& Nicas, T. I. Mechanism of action of oritavancin and related glycopeptide antibiotics. FEMS Microbiol. Rev. 93, 511-532 (2003).

(21) Sun H., Maderazo, E. G. \& Krusell, A. R. Serum protein-binding characteristics of vancomycin. Antimicrob. Agents Chemother. 37, 1132-1136 (1993).

(22) Arhin, F. F. et al. Effect of polysorbate 80 on oritavancin binding to plastic surfaces: implications for susceptility testing. Antimicrob. Agents Chemother. 52, 1597-1603 (2008).

(23) Yung-Chi, C. \& Prusoff, W. H. Relationship between the inhibition constant $\left(K_{3}\right)$ and the concentration of the inhibitor which causes 50 per cent inhibition $\left(\mathrm{I}_{50}\right)$ of an enzymatic reaction. Biochemical Pharmacology 22, 3099-3108 (1973).

(24) Allen, N. E., LeTourneau, D. L. \& Jr. Hobbs, J. N. The role of hydrophobic side chains as determinants of antibacterial activity of semisynthetic glycopeptide antibiotics. J. Antibiotics 50, 677-684 (1997).

(25) Cooper, M. A., Fiorini, M.T., Abell, C. \& Williams, D.H. Binding of vancomycin group antibiotics to D-alanine and D-lactate presenting self-assembled monolayers. Bioorg. Med. Chem. 8, 2609-2616 (2000).

(26) Rao, J., Yan, L., Xu, B. \& Whitesides, G.M. Using surface plasmon resonance to study the binding of vancomycin and its dimer to self-assembled monolayers presenting D-Ala-D-Ala. J. Am. Chem. Soc. 121, 2629-2630 (1999).

(27) Cooper, M. A., Williams, D. H. \& Cho, Y. R. Surface plasmon resonance analysis of glycopeptide antibiotic activity at a model membrane surface. Chem. Commun. 17, 1625-1626 (1997). 
(28) Mercier, R-C., Houlhan, H. H. \& Rybak, J. M. Pharmacodynamic evaluation of a new glycopeptide, LY333328, and in vitro activity against Staphylococcus aureus and enterococcus faecium. Antimicrob. Agents Chemother. 41, 1307-1312 (1997).

(29) McKay, G. A., Beaulieu, S., Lehoux, D., Parr, JR.T. \& Moeck, G. Methods of treating bacterial infections using oritavancin. Patents US 2012/0035097 A1 (2012).

(30) Vuignier, K., Schappler, J., Veuthey, J-L., Carrupt, P-A. \& Martel, S. Drug-protein binding: a critical review of analytical tools. Anal Bioanal Chem 398, 53-66 (2010).

(31) Rotschafer, J. C. et al. Pharmacokinetics of vancomycin: Observations in 28 patients and dosage recommendations. Antimicrob. Agents Chemother. 22, 391-394 (1982).

(32) Bell, G. I. Models for the specific adhesion of cells to cells. Science 200, 618-627 (1978).

(33) Wu, Y. et al. Transforming binding affinities from three dimensions to two with application to cadherin clustering. Nature 475, 510-513 (2011). 


\section{ACKNOWLEDGEMENTS}

We thank the EPSRC Interdisciplinary Research Centre in Nanotechnology (Cambridge, UCL, Bristol - GR/R45680/01), the Royal Society (RS), Bio Nano Consulting (BNC), Targanta Therapeutics, EPSRC Speculative Engineering Program (EP/D505925/1), the European Union FP7 Project VSMMART Nano (managed by BNC), EPSRC Grand Challenge in Nanotechnology for Healthcare (EP/G062064/1) and the EPSRC IRC in Early Warning Sensing Systems for Infectious Diseases (EP/K031953/1) for funding and materials. MAC acknowledges support of a NHMRC Australia Fellowship AF511105. We thank, Samadhan B. Patil, Guillaume T. Charras and Ian K. Robinson for helpful discussions. 


\section{METHODS}

Specificity of drug-target interactions. A strategy of passivation of the 'bottom' side and inline referencing against a control cantilever was adopted to ensure measurements of drugtarget interactions resulted in specific signals only.

Experimental errors. The surface stress data error bars in each set of measurements were determined as the standard deviation of the surface stress data fitted measurements from four separate cantilever chips.

Role of competing soluble ligands. The exact relationship between surface action and the size/nature of the competing ligands was tested by using serum proteins $(67 \mathrm{kDa})$ and diacetyl Lysine-D-Alanine-D-Alanine (Ac-VSR) $(0.37 \mathrm{kDa})$ analogues to bacterial cell wall precursors (Bact. Analog). Comparison between Figs. 3 and 5 show quite similar stress responses for the large and small molecule in solution and the same basic equations (1) and (3) apply to all data. While our measurements demonstrate that the strength of surface drugreceptor binding interactions is not affected by the presence of serum proteins, the drugdiacetyl Lysine-D-Alanine-D-Alanine complexes (or drug-Ac-VSR) in solution phase was observed to interact with the surface bound receptors to induce the stress, driving the enhanced efficacy of surface binding affinity for antibiotics (Fig. 1b). Thus, our results show that in contrast to large-sized competing serum proteins, the pathways labeled by $K_{2}$ and $K_{4}$ in Fig $1 \mathrm{~b}$ for small drug molecules in the presence of small sized strong competing ligands cannot be neglected. In summary, (i) the drug molecules which are not significantly affected by the competing ligands in solution may bind to the surface targets without reducing their surface binding affinities, (ii) a bound drug-target complex in solution may increase the polarisation of a dimer binding pocket and in turn promote surface dimerisation, greatly 
enhancing the binding strength to the surface receptors, and (iii) in the case of weak competing ligands, the binding of a drug molecule to a ligand in solution may block the access of surface active binding sites. We demonstrated these principles by evaluating the modes of action of the clinically important glycopeptide group of antibiotics to reveal how they are affected by the solution matrix. We show that Ori/Ori, Ori/serum, Van/Van and Van/serum solution matrices under physiological conditions yield nanomechanical stress assays which are in good agreement with the drugs' efficacy.

To demonstrate that the model as shown in (ii) can be used to enhance the surface binding interactions and to control the functionality of drug molecules, we implemented this model according to the complex interactions derived from the combinations of Ori/diacetyl LysineD-Alanine-D-Alanine and Van/diacetyl Lysine-D-Alanine-D-Alanine (Supplementary Table S2). We find that in contrast to drug/serum complex, the drug/diacetyl Lysine-D-Alanine-DAlanine complexes of Ori over Van improves surface binding activity 500-fold. Such mechanism is particularly important for drug molecules, where the increased surface binding activity, can lead to increased surface stress to alter the mechanics of bound surface receptors, boosting the efficiency of drugs for killing pathogens. This is a major advance because it means that the efficiency of drugs may be enhanced by administering a combinational therapy of drug/small sized strong competing ligands. Future work will investigate the extent of solution matrices of $\mathbf{O r i} / \mathbf{V a n} / \mathrm{serum}, \boldsymbol{O r i} / \mathbf{V a n} /$ serum/diacetyl Lysine-D-Alanine-D-Alanine, Van/serum/diacetyl Lysine-D-Alanine-D-AlanineandOri/serum/diacetyl Lysine-D-AlanineD-Alanine on cantilevers to test if these combinations can improve the efficacy over $\mathbf{V a n} /$ serum and $\boldsymbol{O r i} /$ serum complexes applicable to the present day clinical practice. 


\section{A Table and Figure captions}

\begin{tabular}{llllll}
\hline Description & Receptor & $\begin{array}{l}\text { Detection } \\
\text { sensitivity } \\
\text { SPR } \\
(\mathrm{nM})\end{array}$ & $\begin{array}{l}\boldsymbol{K}_{\boldsymbol{I}} \\
\mathbf{S P R} \\
(\mu \mathrm{M})\end{array}$ & $\begin{array}{l}\text { Detection } \\
\text { sensitivity } \\
\text { cantilever } \\
(\mathrm{nM})\end{array}$ & $\begin{array}{l}\boldsymbol{K}_{\boldsymbol{I}} \text { Cantilever } \\
(\mu \mathrm{M})\end{array}$ \\
\hline Van & VSR & $\begin{array}{l}310 \\
\text { (ref. 25) } \\
\text { (ref. 26) }\end{array}$ & $\begin{array}{l}1.1 \\
\text { (ref. 26) }\end{array}$ & $\begin{array}{l}10 \\
\text { (ref. 15) }\end{array}$ & $0.5 \pm 0.2$ \\
& & & & \\
Ori & nSR & $\begin{array}{l}0.07 \\
\text { (ref. 27) }\end{array}$ & 0.1 & $0.04 \pm 0.01$ \\
\hline
\end{tabular}

$\mathrm{n} / \mathrm{a} \quad$ SPR value not available

Table 1 The detection sensitivity of drug-target interactions on cantilever arrays compared with the SPR measurements.

Figure 1: Exploiting the nanomechanics of drug-target interactions to investigate the impact of dosing and competing ligands on the functionality of drug molecules; (a) Schematic representation of solution and surface interactions, where molecules can bind to the surface tethered receptors to form a bound complex. The efficacy is measured for a model bacteria cell wall analogue tethered to one side of a cantilever surface. (b) Schematic representation of the overall concept for therapeutic efficacy, where molecules can bind to the surface bound receptors to form a complex or interact with a competing ligand in three dimensional (3D) solution to form drug-target complex while blocking the selective inhibitors from binding to the surface bound receptors. (c) Schematic representation of an array of eight rectangular silicon cantilevers, each measuring $500 \mu \mathrm{m}$ long, $100 \mu \mathrm{m}$ wide and $1 \mu \mathrm{m}$ thick. The bending of all cantilevers is monitored in parallel using time-multiplexed optical beam detection on a single photodetector. The red beam is the laser which is focused at the free end of each cantilever sensor to monitor the cantilever bending deflections. The surface bound receptors are represented by vertical sticks, while the antibiotic drug molecules are shown as blue chemical structural cartoons and the serum protein molecules are represented by the red symbols (circle). (d) Schematic representation of complex blood environment from blood vessel to demonstrate the challenge of real time nanomechanical detection of active free drugs in bloodstream and to investigate the impact of the nature of solvent matrix on the bioactivity of drugs.

Figure 2: The nanomechanics of drug-target interactions in weak competing ligand at clinically relevant concentrations; (a) The differential surfaces stress for Van binding from $600 \mu \mathrm{M}(4 \mathrm{~g} / \mathrm{dL})$ of pure model human serum albumin $(H S A)$ to test the specificity and sensitivity of nanomechanics of drug-target interactions in a complex liquid media such as

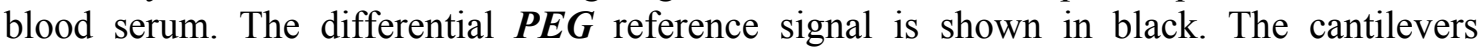
generally bend downwards due to steric and electrostatic repulsive interactions between surface bound drug-target complexes. A negative signal corresponds to a compressive surface 
stress and the downward bending of the cantilever. The greyed-out area represents the injection of serum without the drug for control measurements lasting for 5 mins to establish a baseline. (b) The differential bending signal of $3 \mu \mathrm{M}$ Van in $0 \%$ (green), 50\% (blue) and 98\% (red) blood serum. The corresponding differential PEG reference signals for all three serum concentrations are shown in green, blue and red. The greyed-out area represents the injection of serum without the drug for control measurements lasting for 5 mins to establish a baseline. (c) The corresponding differential bending signals of $0.5 \mu \mathrm{M}$ Ori in $0 \%$ (green), $50 \%$ (blue) and 98\% (red) blood serum. The greyed-out area represents the injection of serum without the drug for control measurements lasting for 5 mins to establish a baseline. (d) The differential surface stress for $3 \mu \mathrm{M}$ Van monitored as a function of model pure human serum albumin (orange) and pooled normal whole human blood serum (NHS) (blue) concentrations diluted in phosphate buffer with PS80 described by the Eq. (1) (solid lines) to calculate $I C_{50}$. The corresponding differential surface stress for $0.5 \mu \mathrm{M}$ Ori monitored as a function of model pure human serum albumin (light blue) and pooled normal whole human serum (NHS) (red) concentrations diluted in phosphate buffer with PS80 described by the Eq. (1) (solid lines) to calculate $I C_{50}$. The surface stress data error bars were determined as the standard deviation of the surface stress data fitted measurements from four separate cantilever chips.

Figure 3: The nanomechanics of drug-target interactions in the presence of strong competing ligand at clinically relevant concentrations; (a) The differential bending signal of $50 \mu \mathrm{M}$ Van in $0 \mu \mathrm{M}$ (green), $50 \mu \mathrm{M}$ (blue) and $500 \mu \mathrm{M}$ (red) (Bact. Analog or Ac-VSR). The differential $\boldsymbol{P E G}$ reference signal is shown in black. The greyed-out area represents the injection of diacetyl Lysine-D-Alanine-D-Alanine (Ac-VSR) analogues to bacterial cell wall precursors (Bact. Analog) without the drug for control measurements lasting for 10 mins to establish a baseline. (b) The corresponding differential bending signals of $0.1 \mu \mathrm{M}$ Ori in $0 \mu \mathrm{M}$ (green), $30 \mu \mathrm{M}$ (light blue) and $500 \mu \mathrm{M}$ (red) (Bact. Analog or Ac-VSR). The greyed-out area represents the injection of diacetyl Lysine-D-Alanine-D-Alanine (Ac-VSR) analogues to bacterial cell wall precursors (Bact. Analog) without the drug for control measurements lasting for 10 mins to establish a baseline. (c) A semi-log plot showing the measured differential stress response for surface bound receptors as a function of diacetyl Lysine-DAlanine-D-Alanine (Bact. Analog or Ac-VSR) concentrations in solution, superimposed on the results of the fit according to Eq. (1) for Van shown by solid diamond symbols in blue. The data are described by Eq. (1) for $K_{1}=0.5 \pm 0.2 \mu \mathrm{M}$ (solid lines, black) and for $K_{1}=0.46$ $\pm 0.02 \mu \mathrm{M}$ (dotted lines, orange). The surface stress data error bars were determined as the standard deviation of the surface stress data fitted measurements from four separate cantilever chips. (d) A semi-log plot showing the measured differential stress response for surface bound receptors as a function of diacetyl Lysine-D-Alanine-D-Alanine concentrations in solution, superimposed on the results of the fit according to Eq. (1) for Ori shown by solid circle symbols in red. The data are described by Eq. (1) for $K_{1}=0.04 \pm 0.1 \mu \mathrm{M}$ (solid lines, black) and for $K_{1}=(9.6 \pm 2.4) \times 10^{-4} \mu \mathrm{M}$ (dotted lines, orange). The surface stress data error bars were determined as the standard deviation of the surface stress data fitted measurements from four separate cantilever chips.

Figure 4: Investigating the mechanics of drug-target interactions using cantilever sensor arrays. The differential bending signals in buffer for $\boldsymbol{V S R}$ signals of $0.1,10,50,100$ and 500 nM Ori respectively. The differential $\boldsymbol{P E} \boldsymbol{G}$ reference signal is shown $\left(\boldsymbol{P E} \boldsymbol{G}_{2}-\boldsymbol{P E} \boldsymbol{G}_{\boldsymbol{I}}\right.$ black). The downward bending signal corresponds to a compressive (repulsive) surface stress and the minimum detectable drug concentration is $100 \mathrm{pM}$, showing that the cantilever differential bending signal scales with the drug load. The greyed-out area represents the injection of 
phosphate buffer solution without the drug for control measurement lasting for 10 mins to establish a baseline.

Figure 5: The nanomechanical quantitation of drug-target interactions in whole blood serum at clinically relevant concentrations; (a) The differential bending signal at $98 \%$ whole blood serum for $1 \mu \mathrm{M}$ Van (orange), $5 \mu \mathrm{M}$ Van (green) and $50 \mu \mathrm{M}$ Van (light blue). The differential PEG reference signal is shown in black. The greyed-out area represents the injection of serum without drug for control measurement lasting for 5 mins to establish a baseline. (b) The corresponding differential bending signals at $98 \%$ whole blood serum for $1.5 \mu \mathrm{M}$ Ori (red), $3 \mu \mathrm{M}$ Ori (blue) and $5 \mu \mathrm{M}$ Ori (green). The differential PEG reference signal is shown in black. The greyed-out area represents the injection of serum without the drug for control measurement lasting for 5 mins to establish a baseline.(c) A semi-log plot showing the measured differential surface stress response for surface bound receptors as a function of drug concentrations in solution, superimposed on the results of the fit according to Model (II) Eq. (3) for Van in buffer (open symbols in blue) where [ligand 2 ] $=0$ and in whole blood serum (solid symbols in blue) where the parameters $\sigma_{\max }, m$, and $K_{1}$ were fixed to their respective values in phosphate buffer while the serum concentration was fixed at $\sim 98 \%$ to calculate $K_{3}$. The surface stress data error bars were determined as the standard deviation of the surface stress data fitted measurements from four separate cantilever chips. (d) A semi-log plot showing the measured differential surface stress response for surface bound receptors as a function of drug concentrations in solution, superimposed on the results of the fit according to Model (II) Eq. (3) for Ori in buffer (open symbols in wine) where [ligand $\left.{ }_{2}\right]=0$ and in whole blood serum (solid symbols in red) where the parameters $\sigma_{\max }, m$, and $K_{1}$ were fixed to their respective values in phosphate buffer while the serum concentration was fixed at $\sim 98 \%$ to calculate $K_{3}$. The surface stress data error bars were determined as the standard deviation of the surface stress data fitted measurements from four separate cantilever chips. 

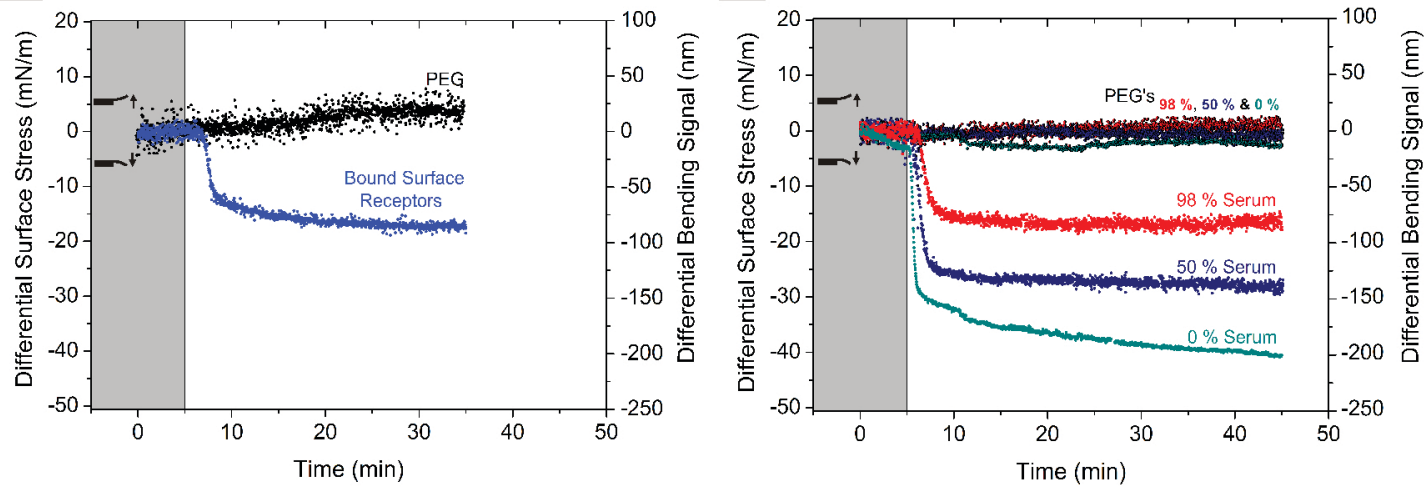

C
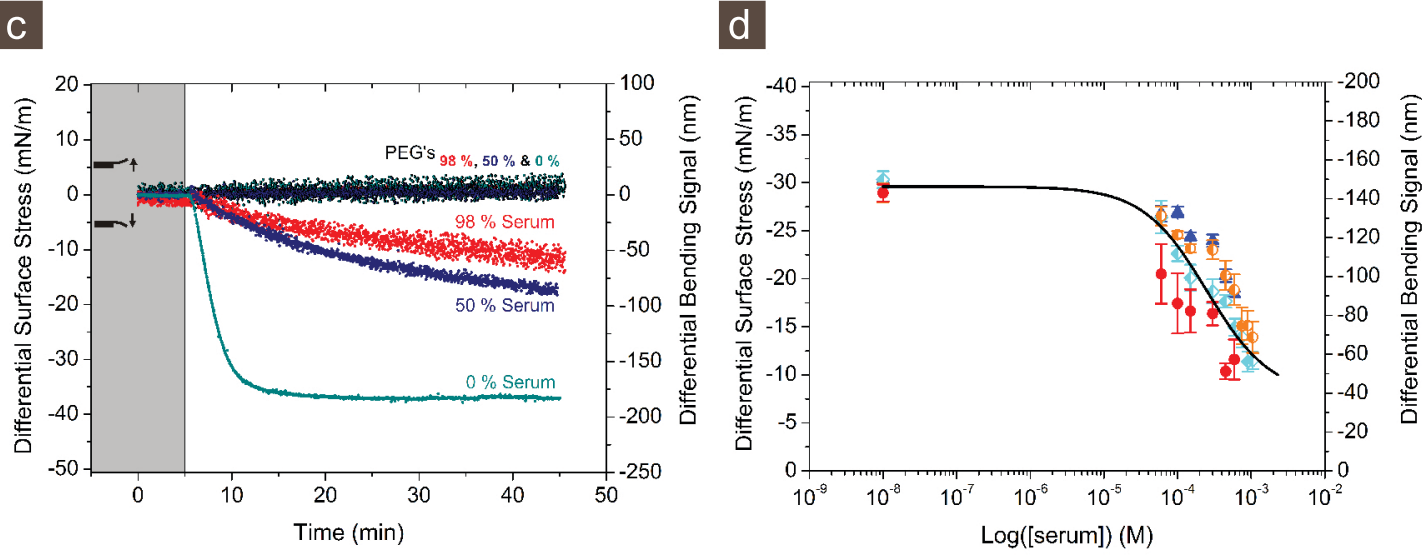

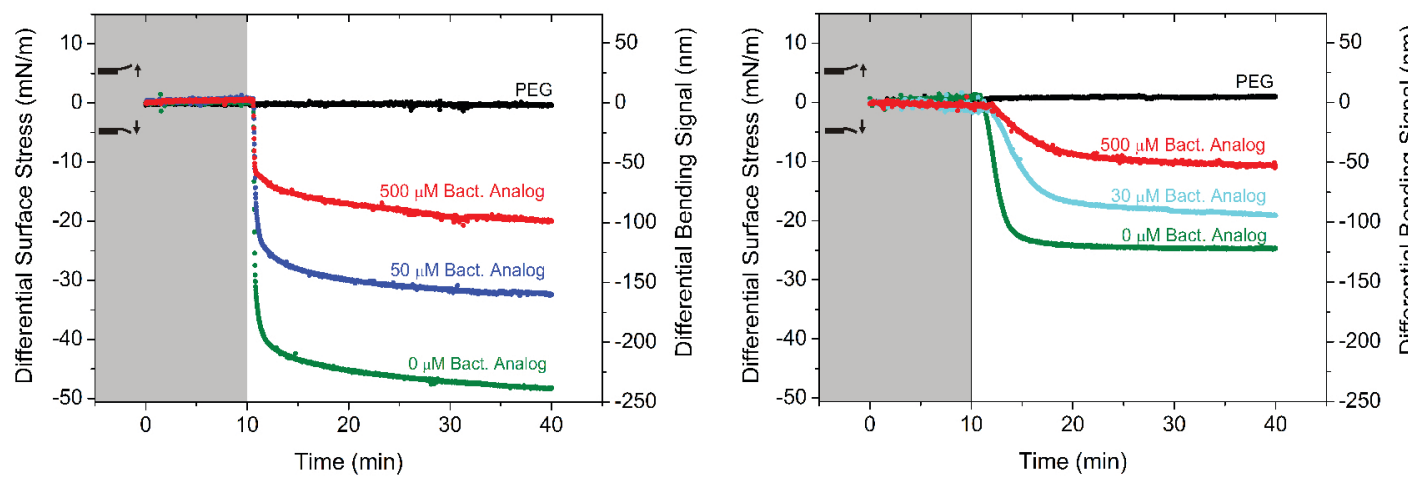

C

d
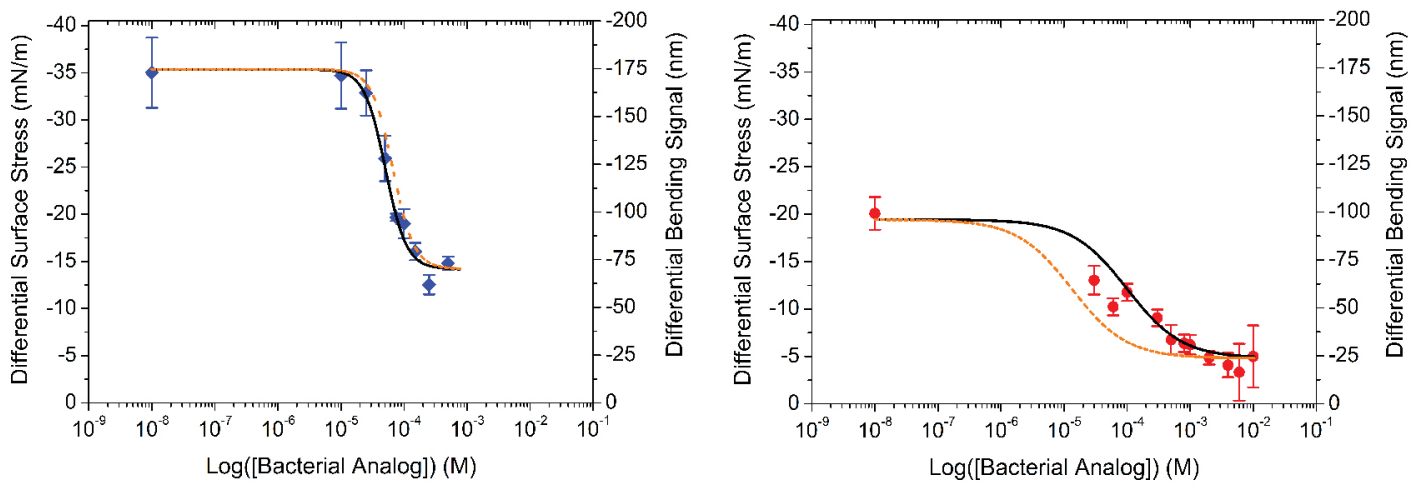


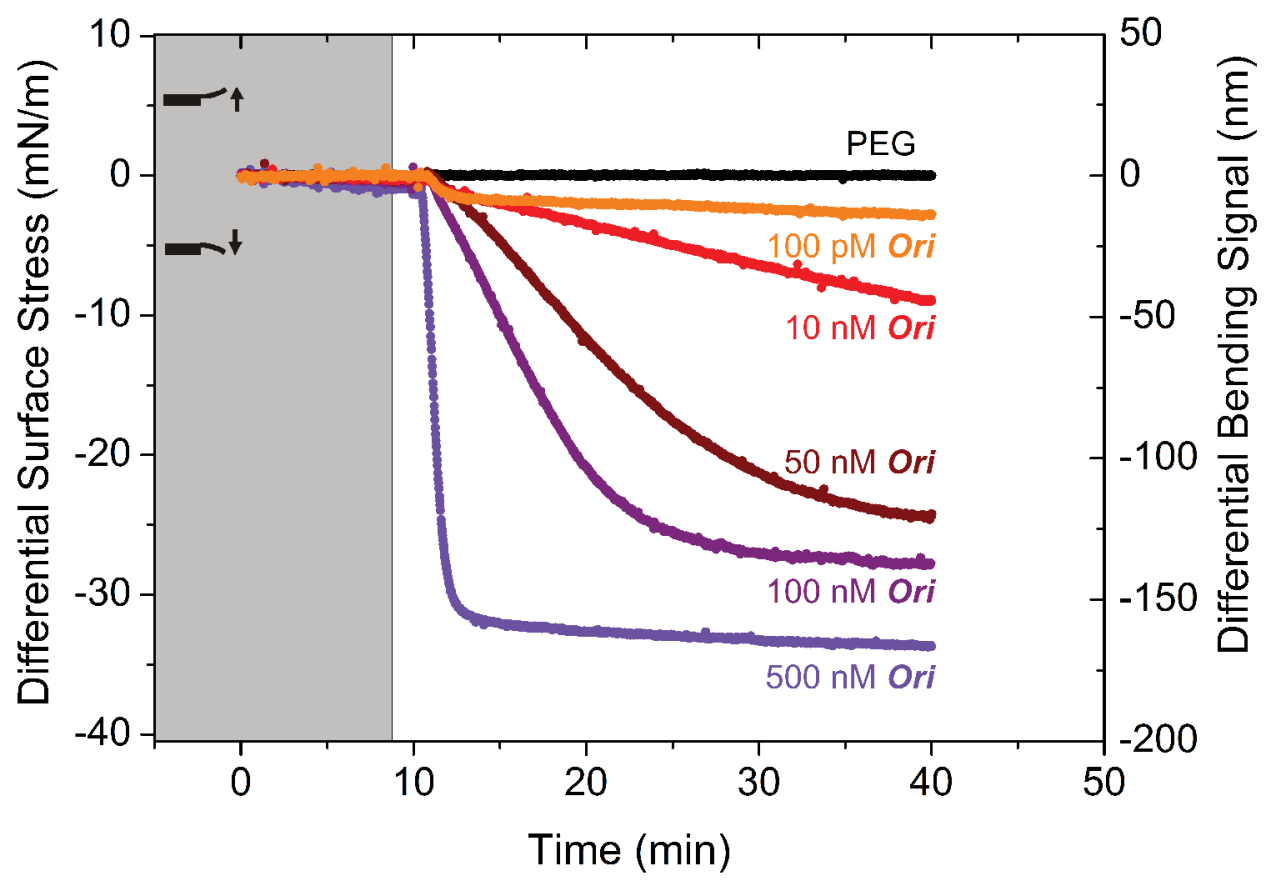


